\title{
Materials Design and Analysis of Low-Power MEMS Microspeaker Using Magnetic Actuation Technology
}

\author{
FATIMAH LINA AYATOLLAHI ${ }^{1, a}$, BURHANUDDIN YEOP MAJLIS ${ }^{1, b}$ \\ ${ }^{1}$ Institute of Microengineering and Nanoelectronics \\ Universiti Kebangsaan Malaysia \\ 43600 Bangi, Selangor, MALAYSIA \\ Email: ${ }^{a}$ lina.ayat@gmail.com, burhan@vlsi.eng.ukm.my
}

Keywords: Hearing Aids, MEMS, Microspeaker, Membrane, Coil, Polyimide, Rare Erath Magnet.

\begin{abstract}
This paper presents material properties of a micromachined mesoscopic acoustic speaker for hearing aid applications. The microspeaker has a single turn copper coil on a polyimide membrane, and an $\mathrm{NdFeB}$ permanent magnet beneath the membrane. The fields due to the permanent magnet were computed using FEMM. Then, forces based on the fields and on the coil driving current were computed. Finally, IntelliSuite was employed to simulate membrane displacements and stresses. The device, with a polyimide membrane diameter of $2.5 \mathrm{~mm}$ and thickness of $2 \mu \mathrm{m}$, consumes $3.4 \mathrm{~mW}$ to generate a sound pressure level of $108 \mathrm{~dB}$ in the human ear.
\end{abstract}

\section{Introduction}

Hearings loss degrades the health of millions of people. A hearing aid is a small electronic device that is worn in or behind the ear. It makes sounds louder, so that a person with hearing loss can listen better and participate more fully in daily activities. The three major components of a hearing aid are the microphone, amplifier and the speaker. The speaker converts the amplified electronic signals to sound waves within the ear canal.

The critical parameters to be chosen to turn this concept into a design include (a) the membrane material, diameter and thickness, (b) the material, radius, width, thickness and current for the coil, (c) the membrane-magnet gap and (d) the magnet material, diameter and length. The geometries and materials for the outer ring and the spacer, and adhesives to hold all parts together, are important practically, but do not directly impact the performance of the speaker.

Concept and Geometry. The concept presented here is based on assembling four small parts in order to build a microspeaker [1]. Fig. 1 is a schematic of the microspeaker assembled from the parts. The key component is a thin flexible membrane to which a single-turn circular coil is bonded. It is held by an outer ring, which also holds a spacer, on the top of which is the strong permanent magnet. Currents through the coil, which vary in time according to the desired sound, produce magnetic fields that interact with the permanent magnetic field to produce forces on the membrane. The forces deflect the membrane, which generates sound. This paper deals with the materials for fabrication and assembly of the microspeaker. 


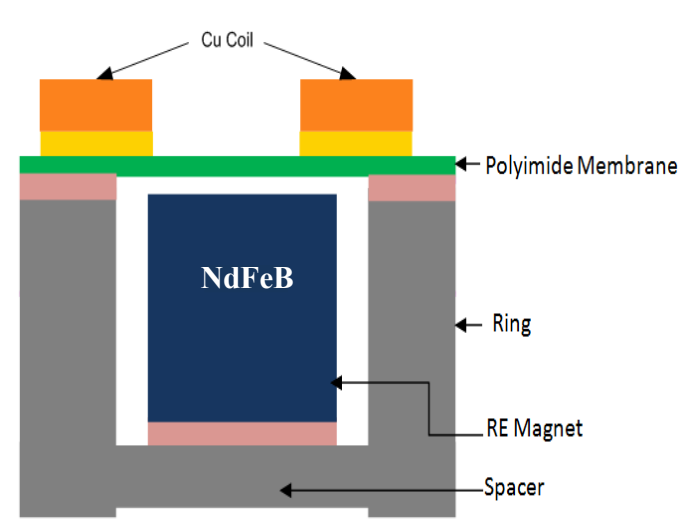

Fig.1 Cross section of the microspeaker showing the four primary components.

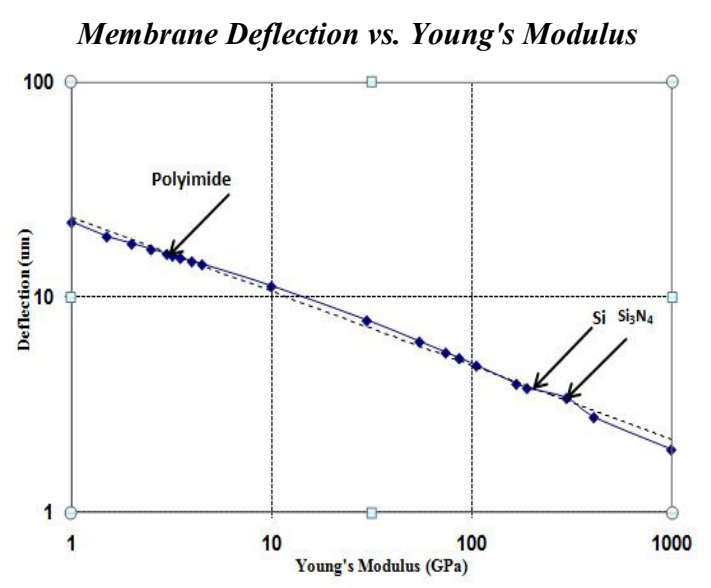

Fig.2 Membrane deflection as a function of Young's Modulus. The dashed line shows the $1 / \mathrm{K}$ trend

Membrane. Polyimides are a very interesting group of incredibly strong and remarkably heat and chemical resistant polymers [2]. When compared to most other organic or polymeric materials, polyimides exhibit an exceptional combination of mechanical toughness thermal stability $\left(>500^{\circ} \mathrm{C}\right)$, and chemical resistance. Because of their high degree of elasticity and inherently low CTE, polyimides can be readily implemented into a variety of microelectronic applications. The choice of polyimide for the membrane material is the result of the set of IntelliSuite simulations using materials of widely varying Young's Modulus (E). Higher Young Modulus means stiffer material and consequently less displacement under the same applied force. The results in Fig. 2 show that polyimide with $\mathrm{E}=3.2 \mathrm{GPa}$ is a very good elastic membrane material. It is also relatively easy to manufacture.

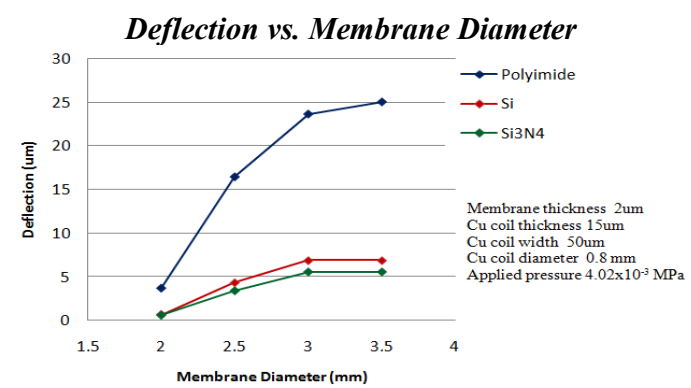

Fig.3 Effect of the Membrane Diameter on the Deflection

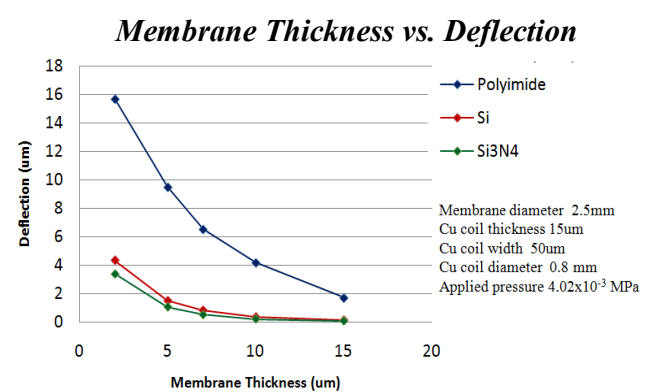

Fig 4 Effect of the Membrane Thickness on the Deflection

Varying the membrane diameter is reasonable in order to get good deflection for small devices. Fig. 3 shows the effect of the diameter on the deflection of the membrane. It shows that increasing the diameter of membrane results on higher diflection, however it is increasing the size of the device. A polyimide diameter of $2.5 \mu \mathrm{m}$ gives a good balance between deflection and device size.

Thickness is one of the important design parameters affecting the device deflection behavior. Maximum thickness of the movable structure is limited by fabrication and the minimum membrane integrity considerations. Fig. 4 illustrated the effect of the thickness on the deflection of the membrane. It is clear that increasing in thickness results a stiffer structure, and will reduces the deflection amount under the same pressure conditions. These results lead to the choice of $2 \mu \mathrm{m}$ for the polyimide thickness. 
Coil. Thick layer of the copper proposed for the one turn coil due to the good conductivity, heat transfer and also easy to electroplate in fabrication process. Coil width is one of the important design parameters affecting the device deflection behavior.

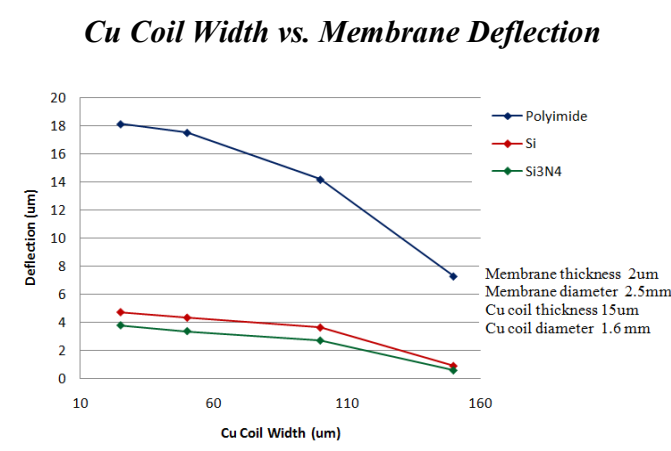

Fig.5 Effect of the Cu Coil Width on the Deflection

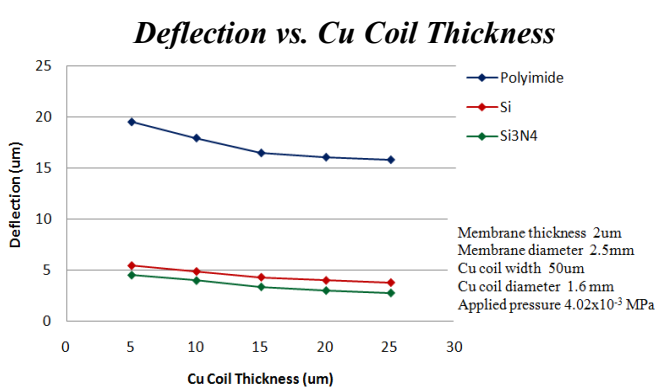

Fig.6 Effect of the Cu Coil Thickness on the Deflection

Effects due to the copper coil width represented in Fig.5. It is observed that, with increasing the width of coil, the deflection of membrane will gradually decreased regardless of the membrane and coil materials. It is due to reduction of area of the membrane that can deflect. However, increasing the width of coil would help lower the power dissipation and heat transfer in the coil. Coil width of $50 \mu \mathrm{m}$ has been chose in this design. The copper coil has a resistivity of $1.7 \times 10^{-6}$ $\Omega \mathrm{cm}$. Fig.6 shows the effect of varying the copper coil thickness on the membrane deflection. It is observed that there is little influence of the coil thickness on the membrane deflection. A thinner coil is easier to make, but has higher resistivity and heating, so the intermediate value of $15 \mu \mathrm{m}$ was picked. A diameter of $1.6 \mathrm{~mm}$ has been chosen for coil due to the diameter of commercial rare-earth magnets. The diameter of magnet is $1.59 \mathrm{~mm}$; the best place for coil is in the edge of the magnet, where the tangential flux density is higher.

Magnet. Design of microspeaker is biased on membrane deflection where the magnet produces a constant field that interacts with the field of the current to produce force. This force will vibrate the membrane and produced sound. Deflection force scales proportional to field strength. Strong neodymium rare earth magnets REFeB has chosen. Neodymium magnets are over 10 times stronger than the strongest ceramic magnets [3].Neodymium magnets are a composition of mostly Neodymium, Iron and Boron. If left exposed to the elements, the iron in the magnet will rust. To protect the magnet from corrosion and to strengthen the brittle magnet material, it is usually preferable for the magnet to be coated. There are a variety of options for coatings, but nickel is the most common and usually preferred. Nickel plated magnets are triple plated with layers of nickel, copper, and nickel again. This triple coating makes magnets much more durable than the more common single nickel plated magnets.

Ring. The function of the ring is to hold the combination of the magnet and spacer in position relative to the membrane with the coil. The ring and spacer are held together by a friction fit. The ring and membrane are held together by an adhesive. The material of the ring could be plastic, ceramic or metal. A non-magnetic alloy tube is required for production of the ring.

Spacer. Different materials could be used in choosing the spacer; they are including plastic, ceramic or metal. Since the function of spacer is to hold the magnet in position relative to the membrane with the coil. Therefore the most convenient materials are metallic rods that are soft magnets, such as iron. Thus magnet is held to the spacer by magnetic attraction. Spacer was attaching to the outer ring which has the membrane attached to it.

Contact Cap. The function of the cap with electrodes is to provide electrical contacts to the coil leads and to the circuit in which the speaker is used. The cap will consist of a cylindrical plastic 
piece embedding two metallic electrodes. A wide variety of plastics can be used for the cap, for example, polycarbonate or polyethylene. The electrodes will be made of a metallic alloy $0.5+/-$ 0.005 millimeters thick. The alloy must not corrode in normal clean air to more than 1 nanometer in 1 day. The alloy must have a composition that permits bonding of lead wires to it. The electrodes will be punched out of a metal strip by the roll-to-roll method. Electrical leads from the electrodes on the contact cap to the circuit that supplies the excitation current will be wire bonded from those electrodes to a small printed circuit board on which the speaker will be mounted [4].

Conclusion. Based on the results above, a compact and low-power MEMS microspeaker for hearing aids using magnetic actuation technology appears feasible. The displacements are linear with input current for this simple single-turn device. The microspeaker requires a very low current in the range of 100 milliamperes. The designed device consumes $3.4 \mathrm{~mW}$ of the battery power. The small membrane size and deflections is the device we envision would not produce useful sound levels in open air. But, in the confines of an ear, where there is an air volume of roughly 2 cubic centimeters, the deflections are sufficient to achieve practical levels of sound [5]. Sound Power Level (SPL) is defined relative to the threshold of hearing without a hearing aid, which is 20 microPascals. Using the results of the simulations for a $100 \mathrm{~mA}$ current, we compute SPL of 108 $\mathrm{dB}$ within the ear. Material properties for micro-machined mesoscopic acoustic speaker have been performed. The chosen materials represent balances between competing factors, for example, membrane thickness and fabrication processes limit. The next steps in this project are to produce and test the design based on the materials reported above.

\section{References}

[1] Fatima Lina Ayatollahi and Burhanuddin Yeop Majlis, Design and Modeling of Micromachined Condenser MEMS Loudspeaker Based on Silicon Membrane Using Permanent Magnet (Nd-Fe-B), Proc. RSM 2007, Penang, Malaysia, pp. $472-478$.

[2] C. Shearwood, M. A. Harradine, T. S. Birch, and J. C. Stevens, "Applications Of Polyimide Membrane To MEMS Technology,” Microelectron. Eng., vol.30, pp. 547-550, 1996.

[3] K \& J Magnetic Inc., http://www.kjmagnetics.com/

[4] Man-Dae han and Bok-Beum Kim, "Micro Speaker" US Patent 6553126 April 22nd 2003.

[5] M. Cheng, W. Huang, and S. R. Huang, "A Silicon Microspeaker For Hearing Instruments," J. Micromech. Microeng., vol. 14, no. 7, pp. 859-866, Jul. 2004. 